\title{
Study on the optimal design of long-distance branch distribution network in rural drinking water safety project WANG Wenfen ${ }^{a}$, CHEN Chen ${ }^{b}$
}

School of Construction Equipment and Municipal Engineering, JiangSu JianZhu Institute

No.26 Xueyuan Road,Xuzhou,221000,China

awangwenfen1987@126.cn, b150103294@qq.cn

Keywords: branch distribution network; optimal design; coupling constraint; linear programme Abstract. On the basis of the traditional linear programming model,this paper presents a optimal design mathematical model of gravity branch distribution network. Considering the coupling constraint, the model takes the length of main and subordinate pipe having standard diameter as decision variables. The minimum works investment is taken as objective variable, and the MATLAB is used to solve. There are a better result in the optimal design of branch distribution network with this method in the application cases, and which can provide a basis for the rural drinking water safety project.

\section{Introduction}

The branch pipe network is used widely in the irrigation and water supply systems, and researchers at home and abroad have been paying great attention to the optimization design problems. Karmeli ${ }^{[3]} 、$ Gupta $^{[4,5]}$ etc. has presented the linear programming model, which can get the global optimal solution. And th model has been applied by WEI Yongyao ${ }^{[6,7]}$, BAI Dan ${ }^{[8]}$ etc. The purpose of the optimal design is to satisfy the desired pressure and flow of each node, to seek the most economical design. This paper presents a optimal design mathematical model of gravity branch distribution network.

\section{Establishment of the model of branch distribution network}

For the gravity branch distribution network which supply the water by the gravity flow, there is no need to set the pump station. The investment is mainly composed of the pipe network cost. The purpose of the optimal design is to satisfy the desired pressure and flow of each node, to select the size of the pipe to get the minimum investment ${ }^{[9]}$.

\section{Objective variable}

At first,we divide the main pipe of the branch pipe network in Fig. 1 into $N$; then we define the total length of main pipe as $l_{g i}$, subordinate pipe as $l_{z i}$, and take the length of main and subordinate pipe having standard diameter $X_{i j} 、 X_{i, j+N_{b}}$ as decision variables, the minimum works investment as objective variable. 


$$
\min W=\sum_{i=1}^{N}\left(\sum_{j=1}^{N_{g b}} C_{i j} X_{i j}+\sum_{j=1}^{N_{z b}} C_{i, j+N_{g b}} X_{i, j+N_{g b}}\right)
$$

Where: $W$ (yuan) stands for the investment of the gravity branch distribution network; $N_{g b \text { 、 }}$ $N_{z b}$ respectively stand for the number of each main and subordinate pipe having standard diameter; $C_{i j} 、 X_{i j}$ respectively stand for the unit price $(\mathrm{yuan} / \mathrm{m})$ and the length $(\mathrm{m})$ of the $\mathrm{j}$-th standard diameter in the i-th main pipe; $C_{i, j+N_{g b}} 、 X_{i, j+N_{g b}}$ respectively stand for the unit price $(\mathrm{yuan} / \mathrm{m})$ and the length(m) of the subordinate pipe in the i-th main pipe.

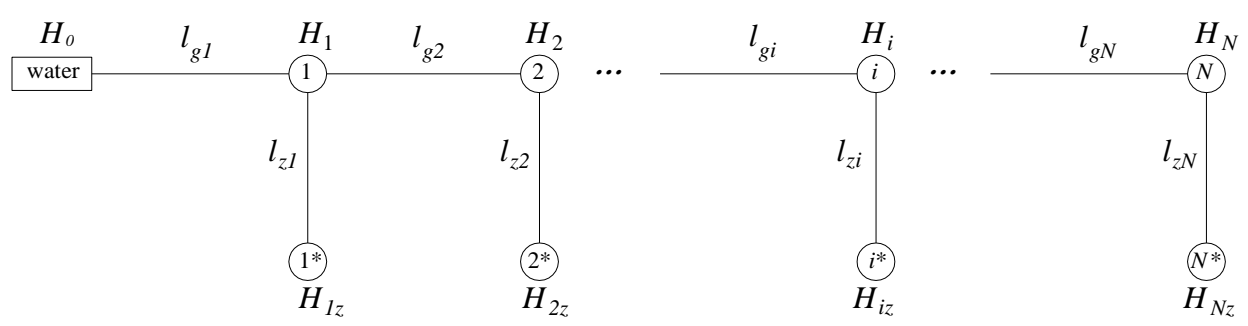

Fig. 1 A simple figure of branch pipe network

\section{Constraint condition}

(1) Constraint condition of length

$$
\begin{aligned}
& \sum_{j=1}^{N_{g b}} X_{i j}=l_{g i} \quad(i=1,2, \cdots, N) \\
& \sum_{j=1}^{N_{z b}} X_{i, j+N_{g b}}=l_{z i} \quad(i=1,2, \cdots, N)
\end{aligned}
$$

(2) Constraint condition of nodal head

$H_{i}$ (the head of the intersections of main and subordinate pipe), $H_{i z}$ (the head of the end of subordinate pipe) should satisfy the follow conditions:

$$
H_{k \min } \leq H_{k} \leq H_{k \max } \quad(k=i, i z ; i=1,2, \cdots, N)
$$

Where: $H_{k \min }(\mathrm{m})$ stands for the minimum allowable head of the note $k$, which can be expressed as follows: $H_{k \min }=Z_{k}+H_{u k}$ while the note $k$ is uesful note, otherwise $H_{k \min }=Z_{k}$; 
$Z_{k}(\mathrm{~m}) 、 H_{u k}(\mathrm{~m})$ respectively stand for the groud elevation and the service head; $H_{k \max }(\mathrm{m})$ stands for the maximum allowable head,which can be expressed as follows: $H_{k \max }=Z_{k}+P_{k \max } ; P_{k \max }(\mathrm{m})$ stands for the maximum allowable pressure of the pipe.

And there also has:

$$
\begin{aligned}
& H_{i}=H_{i-1}-\sum_{j=1}^{N_{g b}} J_{i j} X_{i j} \\
& H_{i z}=H_{i}-\sum_{j=1}^{N_{z b}} J_{i, j+N_{g b}} X_{i, j+N_{g b}}
\end{aligned}
$$

Then put formula(5) (7) into formula (4), we can gain:

$$
\begin{aligned}
& H_{i-1}-Z_{i}-P_{i \max } \leq \sum_{j=1}^{N_{g b}} J_{i j} X_{i j} \leq H_{i-1}-Z_{i}-H_{u i} \\
& H_{i-1}-Z_{i z}-P_{i z \max } \leq \sum_{j=1}^{N_{g b}} J_{i j} X_{i j}-\sum_{j=1}^{N_{z b}} J_{i, j+N_{g b}} X_{i, j+N_{g b}} \leq H_{i-1}-Z_{i z}-H_{u i z}
\end{aligned}
$$

Where: $J_{i j}$ stands for the hydraulic gradient of the $\mathrm{j}$-th standard diameter in the $\mathrm{i}$-th main pipe according to the Pavlov formula, $J_{i j}=10.29 \alpha n^{2} \frac{Q_{i j}^{2}}{D_{i j}^{5.33}} ; \alpha$ stands for the amplification factor considering the local head loss; $n$ stands for the pipe roughness coefficient; $Q_{i j}\left(\mathrm{~m}^{3} / \mathrm{h}\right)$ stands for the flow of the ij-th pipe $D_{i j}(\mathrm{~m})$ stands for the $\mathrm{j}$-th standard diameter in the i-th main pipe; $J_{i, j+N_{g b}}$ stands for the hydraulic gradient of the subordinate pipe in the i-th main pipe.

(3) Coupling constraint condition of the system(constraint condition of the water levels at the head and end of the main pipe)

$$
Z_{N}+P_{N \max }-H_{0} \leq \sum_{i=1}^{N} \sum_{j=1}^{N_{g b}} J_{i j} X_{i j} \leq Z_{N}+H_{u N}-H_{0}
$$


(4) Nonnegative constraints

$$
\begin{aligned}
& X_{i j} \geq 0 \quad\left(i=1,2, \cdots, N ; j=1,2, \cdots, N_{g b}\right) \\
& X_{i, j+N_{g b}} \geq 0 \quad\left(i=1,2, \cdots, N_{j} ; j=1,2, \cdots, N_{z b}\right)
\end{aligned}
$$

\section{Solution of model}

The formula (1) (3) and formula (8) (12) constitute a mathematic model of the gravity branch distribution network, and the model has two coupling constraints. The steps of the solution are as follows:

Step1: Divide the pipe into some sections;

Step2: Select standard diameter meeting the flow rate constraints, then bring them into the model calculation;

Step3: Substituting the parameters into the objective function and constraints, solving the linear model to obtain the quadratic optimization solution.

\section{Application example}

Solving the example used by Bai using the optimization method in this paper [9]. The pipe network is shown in Fig.2. In the linear programming model, there are a total of 25 variables (the length of each pipe section has standard diameter), 29 constraints (9 equality constraints-constraint condition of length, 20 inequality constraints-constraint condition of nodal head). Substituting the parameters and using MATLAB to solve the model. The optimal value is given in Table 1.

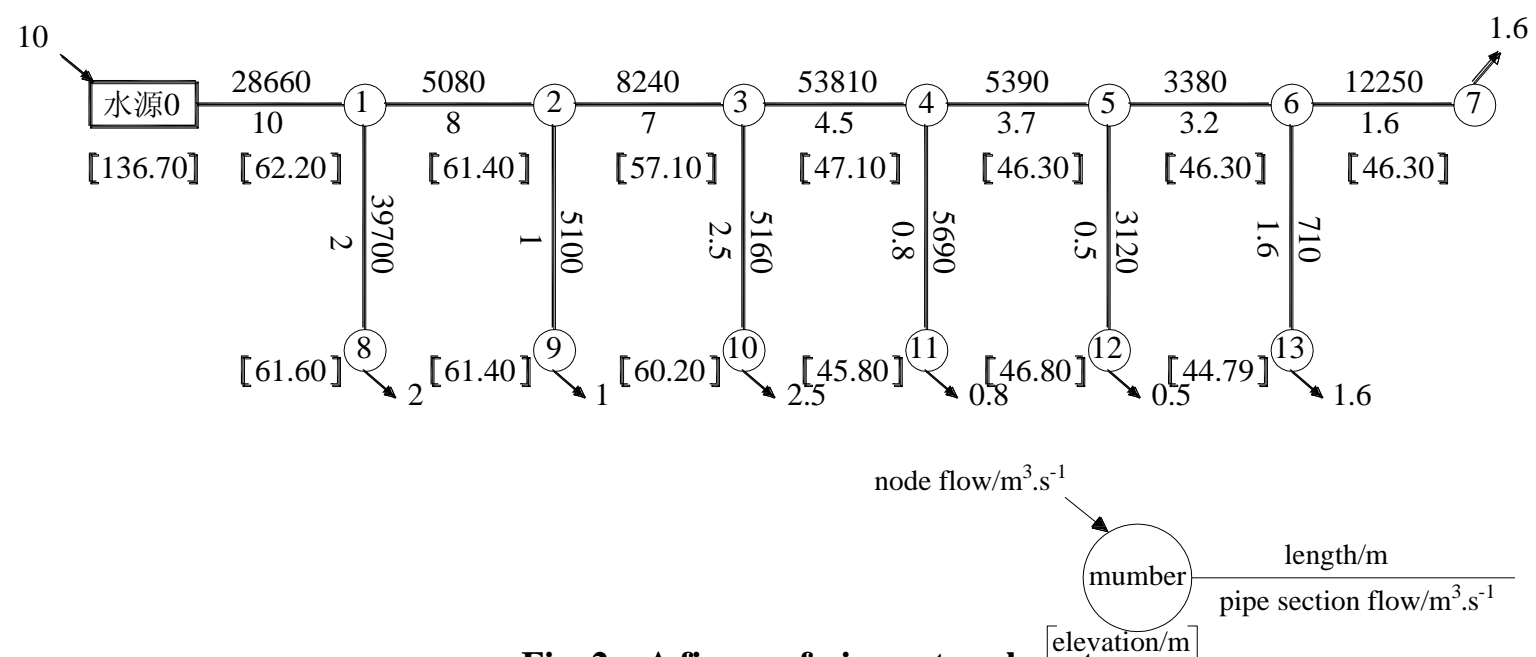

Fig. 2 A figure of pipe network system 
Table 1 optimization results of pipe network system

\begin{tabular}{c|c|c|c}
\hline $\begin{array}{c}\text { Pipe section } \\
\text { number }\end{array}$ & $\begin{array}{c}\text { The name of pipe } \\
\text { section }\end{array}$ & $\begin{array}{c}\text { Selection of pipe } \\
\text { diameter[m] }\end{array}$ & length[m] \\
\hline 1 & water-node 1 & 2.60 & 28660 \\
\hline 2 & node 1- node 2 & $2.60 / 2.80$ & $23865 / 15835$ \\
\hline 3 & node 2- node 3 & 2.40 & 5080 \\
\hline 4 & node 3- node 4 & 2.00 & 5100 \\
\hline 5 & node 4- node 5 & 1.80 & 8240 \\
\hline 6 & node 5- node 6 & 1.80 & 5160 \\
\hline 7 & node 6- node 7 & $1.40 / 1.60$ & $2130 / 51680$ \\
\hline 8 & node 1-node 8 & $1.40 / 1.60$ & 5390 \\
\hline 9 & node 2-node 9 & 1.00 & $438 / 2682$ \\
\hline 10 & node 3-node 10 & $1.20 / 1.40$ & 3380 \\
\hline 11 & node 4-node 11 & 1.00 & 710 \\
\hline 12 & node 5-node 12 & 0.80 & 12250 \\
\hline 13 & node 6-node 13 & 1.20 & \\
\hline
\end{tabular}

\section{Conclusion}

The optimal design mathematical model of gravity branch distribution network is presented in this paper. In the model, the coupling constraint is considered. By the above, we can see that the method in this paper is a feasible and effective method for the optimal design of branch distribution network in the rural drinking water safety project, and also has certain reference value to other works.

\section{References}

[1]Xushi Yan, Suiqing Liu, et al. Water and wastewater pipe network system(2nd edition). Beijing: China Architecture \& Building Press(2008). In Chinese

[2]Lei Chen. China Water Resources(2011)

[3]KarmeliD, GadishY, Meyers S. Journal of Pipeline, ASCE(968)

[4]Gupta I. Trans.Amer.Inst.Ind， Eng(1969)

[5]Gupta I assan M， Cook J. Trans.Amer.Inst， Eng(1972)

[6]Yongyao Wei. Water Saving Irrigation(1983)

[7]Yongyao Wei, Xuezhen Wang. Journal of Hydraulic Engineering(1992)

[8]Dan Bai. Journal of Hydraulic Engineering(1996)

[9]Xuenong Yi, Qun Ren, Guohua Wang, et al. Water and wastewater pipe network engineering design optimization and operation management. Beijing: Chemical Industry Press(2007). In 
Chinese

[10]Meilian Zhang. Journal of Jimei University(2001)

[11]Jilin Cheng. Theory and application of the optimal experimental method for large-scale systems. Shanghai science and Technology Press(2002)

[12]Jilin Cheng, Yuanyu Guo, et al. Acta Mathematica Scientia(2004)

[13]Wenxun Xing, Jinxing Xie. Modern optimization method(2nd edition) . Beijing: Qinghua University press(2005). In Chinese

[14]Rongmin Zhou, Changnan Wang, Yue Yan. Henan Science(2010)

[15]Hossein M V, Alireza M. J.Hydraul, Eng(2006)

[16]Siew C, Tanyimboh TT. Penalty-Free Feasibility Boundary Convergent Multi-Objective Evolutionary Algorithm for the Optimization of Water Distribution Systems. Water Resources Management(2012)

[17]Dong XL, Liu SQ, Tao T. A comparative study of differential evolution and genetic algorithms for optimizing the design of water distribution systems. Journal of Zhejiang University- Science $\mathrm{A}(2012)$ 